Portland State University

PDXScholar

$12-2-2014$

\title{
Charge on Luminous Bodies Resembling Natural Ball Lightning Produced Via Electrical Arcs Through Lump Silicon
}

\author{
Christina L. Porter \\ Reed College \\ Galen P. Miley \\ Reed College \\ David J. Griffiths \\ Reed College \\ Erik J. Sánchez \\ Portland State University, esanchez@pdx.edu
}

Follow this and additional works at: https://pdxscholar.library.pdx.edu/phy_fac

Part of the Physics Commons

Let us know how access to this document benefits you.

\section{Citation Details}

Porter, C. L., Miley, G. P., Griffiths, D. J., \& Sánchez, E. (2014). Charge on luminous bodies resembling natural ball lightning produced via electrical arcs through lump silicon. Physical Review E, 90(6), 063102.

This Article is brought to you for free and open access. It has been accepted for inclusion in Physics Faculty Publications and Presentations by an authorized administrator of PDXScholar. Please contact us if we can make this document more accessible: pdxscholar@pdx.edu. 


\title{
Charge on luminous bodies resembling natural ball lightning produced via electrical arcs through lump silicon
}

\author{
Christina L. Porter, ${ }^{*}$ Galen P. Miley, and David J. Griffiths ${ }^{\dagger}$ \\ Department of Physics, Reed College, Portland, Oregon 97202, USA \\ Erik Sánchez \\ Department of Physics, Portland State University, Portland, Oregon 97201, USA
}

(Received 27 October 2013; published 2 December 2014)

\begin{abstract}
A phenomenon resembling natural ball lightning can be produced via electrical arcing through silicon. We use lump silicon instead of silicon wafers to achieve higher production rates and larger, longer-lived luminous balls than previously reported. The luminous balls consist of a silicon core surrounded by a porous network of loosely bound silicon dioxide nanoparticles. We find that the balls carry a small net charge on the order of $10^{-12} \mathrm{C}$ and propose that the nanoparticles are electrostatically bound to the core due to this charge.
\end{abstract}

DOI: 10.1103/PhysRevE.90.063102

PACS number(s): $52.80 . \mathrm{Mg}, 92.60 . \mathrm{Pw}$

\section{INTRODUCTION}

Sightings of the phenomenon known as ball lightning have been recorded for over a thousand years and compiled by many authors [1-7]. Ball lightning is commonly reported as occurring during thunderstorms in which cloud-to-ground lightning is observed, often immediately after such a strike. It is typically described as a spherical, luminous body with an average diameter of $20-30 \mathrm{~cm}$, traveling along some trajectory in the air for about $1-5 \mathrm{~s}[1,2]$. Natural ball lightning has been described as behaving strangely near conductors or in electric fields [2,7]. Many theories have been proposed as explanations of ball lightning, including plasma, microwave, nuclear, and chemical models $[1,2]$.

We present support for a chemical model first proposed by Abrahamson and Dinniss [8] and refined by several groups [9-14]. The evolution of the model is described in [15]. Abrahamson and Dinniss [8] suggested that a lightning discharge in soil or tree roots could reduce silica to elemental silicon, which would then burn, producing porous networks of oxidizing silicon nanoparticles (NPs). Abrahamson expanded this theory to include the oxidation of other metals such as aluminum [16]. Paiva et al. produced small luminous balls (abbreviated LBs) by sending an arc discharge through high purity silicon wafers [9]. They calculated that the energy density of the objects is reasonable for natural ball lightning [12]. Stephan and Massey measured the temperature of LBs to be $2800-3400 \mathrm{~K}$ and suggested that the luminous objects they produced were sustained by the burning of macroscopic silicon droplets [10]. Ito et al. suggested that a macroscopic silicon core burns, producing an NP "halo" around the core that gives the LB its buoyancy [11]. Ito and Cappelli confirmed this structure with microscopy in 2012 [14]. The key features of the theory, as it stands, are illustrated in Fig. 1. Hill et al. scaled up the reaction using triggered natural lightning, with some success [13].

\footnotetext{
*cporter@alumni.reed.edu

${ }^{\dagger}$ griffith@ reed.edu

łesanchez@pdx.edu
}

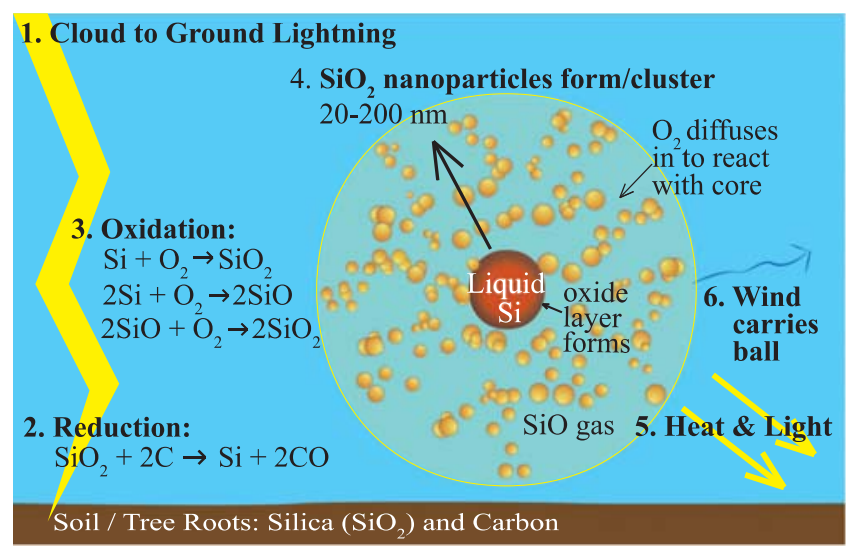

FIG. 1. (Color online) Illustration of the silicon ball lightning model.

Though there are likely several types of ball lightning (not all of which can be explained by the silicon model), a recent natural sighting by Cen, Yuan, and Xue [7] suggests that the silicon model explains at least some natural ball lightning.

In this work, we investigate the structure and composition of silicon LBs produced by arcing through lump silicon. Next, we measure a net charge on LBs by passing them through the electric field of a large parallel plate capacitor, and propose electrostatic attraction as a mechanism for the binding of the observed NP network to the core. We suggest that this charge could account for sightings in which fireballs move with trajectories different from the direction of the wind.

\section{APPARATUS AND PRODUCTION}

The current source used in our experiments is an ac shielded metal-arc welding machine, operated at $30 \mathrm{~V}$ with a current output of $150 \mathrm{~A}$. The work lead of the welder is attached to the copper electrode shown in Fig. 2. The other welder lead 


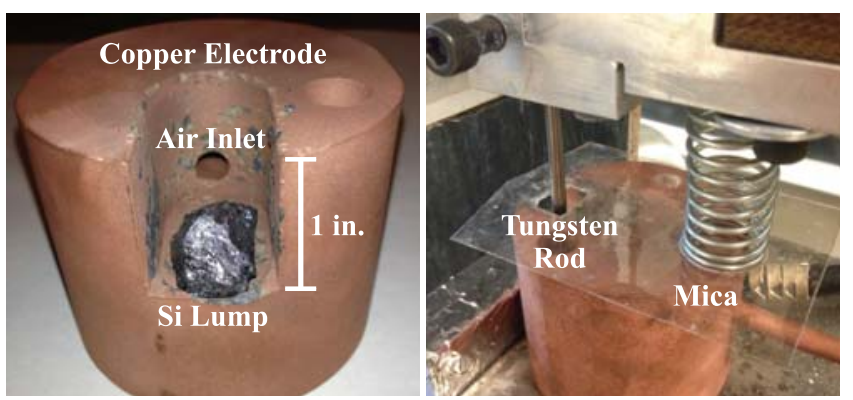

FIG. 2. (Color online) Electrode setup used to maximize production.

is attached to a tungsten electrode (4-mm diameter) mounted into a drill press to allow controlled motion of the tip. A piece of lump silicon ( $98 \%$ purity) is placed in the copper electrode as indicated. A mica sheet is used as a lid for the electrode. To produce LBs, the tungsten rod is tapped against the lump repeatedly, heating it to white hot and drawing an arc with about a half-second duration.

Using lump silicon instead of ultra high purity silicon wafers dramatically increases the production of LBs and the LBs produced have on average longer lifespans and larger diameters. To further increase production, we added an air inlet to the copper electrode into which we pump compressed air that blows over the silicon and toward the observation chamber. The airflow promotes the formation of LBs; conversely, filling the observation chamber with $\mathrm{N}_{2}$ gas extinguishes the LBs, confirming that they are sustained by an oxidation reaction.

With reaction parameters optimized, we produce about 30-100 LBs in each arc [Figs. 3(b) and 3(c)]. The average lifespan of the LBs is $1-2 \mathrm{~s}$, but we frequently see large LBs live for up to $15-20 \mathrm{~s}$. On a CCD camera subject to pixel blooming, the apparent diameter of the LBs we produce is up to $5 \mathrm{~cm}$ [Fig. 3(b)]. By comparison, previous experimenters [11] have reported maximum apparent diameters of $4 \mathrm{~mm}$, and substantially lower production rates. Filtered images show that the actual diameter of a core is up to $4 \mathrm{~mm}$ and the diameter of the surrounding NP network is up to $2 \mathrm{~cm}$ [see Figs. 3(a) and 3(c)].

Our LBs are low density compared to liquid silicon (see [15] and [17]), as first noted in [14]. Our largest LBs can
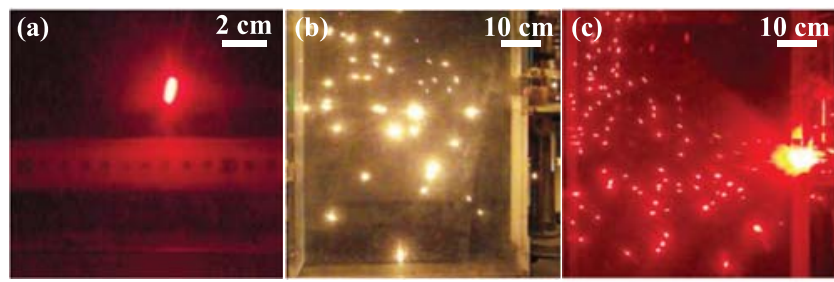

FIG. 3. (Color online) Images taken with a CCD camera of our LBs. (a) and (c) are taken using a 600-nm cutoff filter that shows the distinction between the core and the NPs. Image (b) is unfiltered and shows pixel blooming due to the brightness of the LBs. be convected upward in a wind chamber with an airflow of $5.2 \mathrm{~m} / \mathrm{s}$, a wind speed reasonable for a thunderstorm.

\section{STRUCTURE AND COMPOSITION}

The LBs can be quenched (i.e., prematurely extinguished) upon contact with a surface, leaving a visible core and surrounding flattened NP halo deposited on the surface, as described in [14]. We found that quenching can be achieved to various degrees on many substrates.

We quenched an LB on a silicon wafer and imaged the core and NP halo with a scanning electron microscope (as in [14]). The NPs were imaged moving outward from the core [see Figs. 4(d)-4(1)]. The low magnification optical microscope image in Fig. 4(a) defines the NP imaging locations, and also shows two distinct rings in the halo. The NPs increase in size as you move away from the core. There are two distinct regimes of NP size. Within the inner ring [locations (d)-(f)], a dense network of small NPs (under $50 \mathrm{~nm}$ ) exists. In the outer ring [locations (i)-(k)] the particles are larger $(200-300 \mathrm{~nm})$ and the density of particles is lower. There is a boundary region [images $(\mathrm{g})$ and $(\mathrm{h})$ ] with a mixture of the two regimes. We hypothesize that the inner ring corresponds to the radius past which $\mathrm{SiO}$ vapor concentration significantly drops due to oxidation into $\mathrm{SiO}_{2}$. The turbulent $\mathrm{SiO}$ vapor may prevent particle agglomeration within the inner ring.

We performed energy dispersive spectroscopy (EDS) on a bisected LB core, revealing the structure and composition of both the surface and interior. The interior of the core appears smooth with a finer crystal network than lump silicon. There is a thin oxide layer on the surface of the core, and the interior is metallic silicon, with small traces of copper (from the base electrode).

We also performed EDS and elemental mapping on NPs from the halo of an LB quenched on an aluminum plate, as shown in Fig. 5. Red pixels show oxygen, green silicon, and blue aluminum. The NP region of the elemental map has a fine mixture of red and green pixels, which appear yellow (color online, light gray in print). The elemental map in conjunction with the approximately $2: 1$ ratio of the oxygen and silicon peak heights strongly suggest that the NPs are indeed $\mathrm{SiO}_{2}[18]$.

\section{CHARGE}

We investigated the behavior of LBs in the electric field of a large parallel plate capacitor. Two aluminum plates $(60 \times 20 \mathrm{~cm})$ were charged to $45 \pm 5 \mathrm{kV}$ with a separation distance of $6.5 \mathrm{~cm}$, creating a uniform horizontal electric field $(690 \mathrm{kV} / \mathrm{m})$. LBs fell between the plates from a height of 50 $\mathrm{cm}$ onto a plexiglass floor.

We tracked LBs traveling between the plates with the field pointed, respectively, left, right, and turned off (see video [17]). The results of these analyses are shown in Table I. When the field was turned on in either direction, the vast majority of the LBs entering the field quenched on one of the aluminum walls, with significantly more LBs extinguishing on the positive plate in both cases. When the field was off, a majority of the LBs were extinguished while bouncing on the plexiglass floor, with 


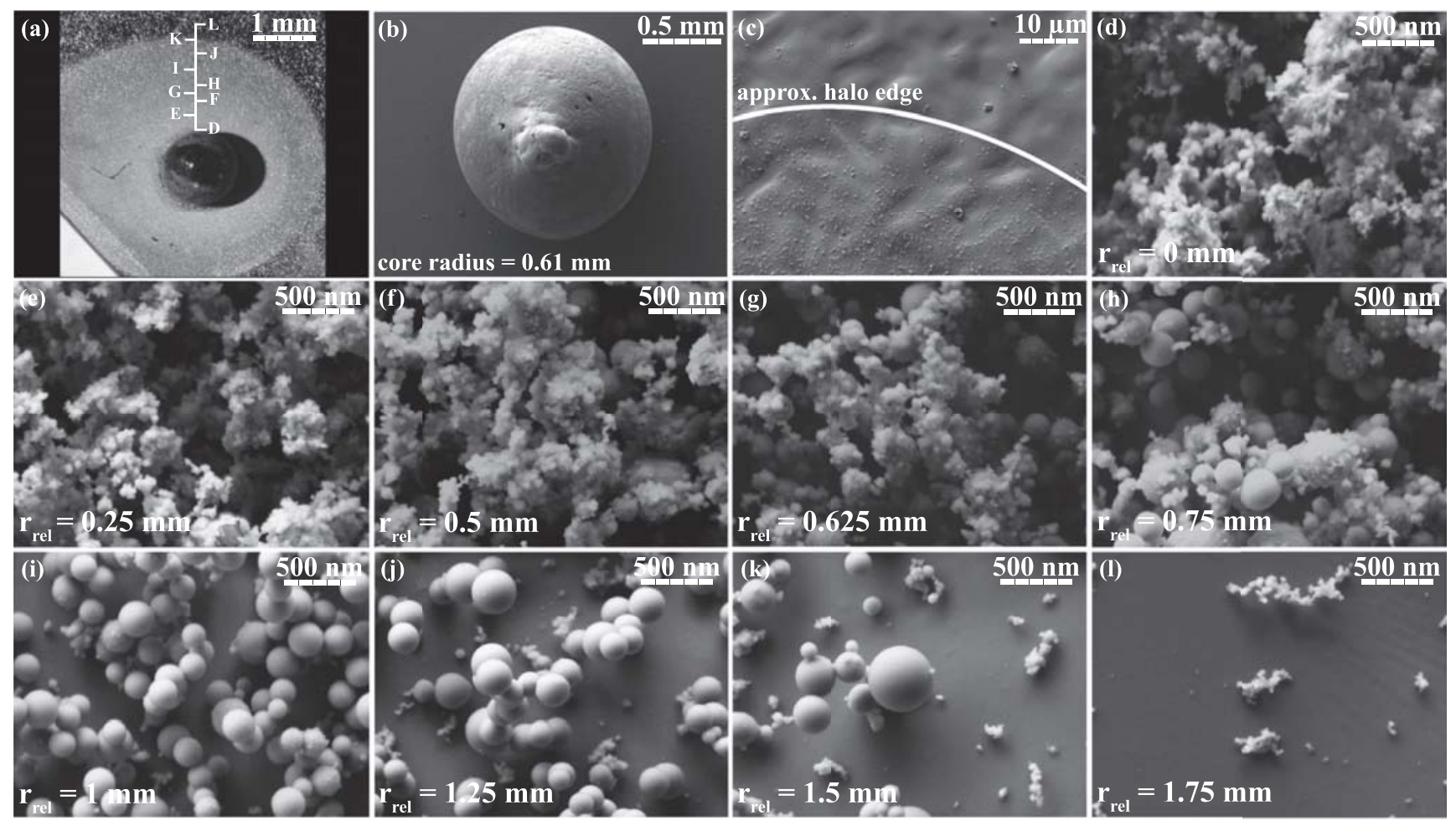

FIG. 4. SEM images of an LB core (b) and NP halo (c-k), quenched on a Si wafer. Halo images are taken along the radius shown in optical microscope image (a) at the specified locations. The value of $r_{\text {rel }}$ in each image indicates the distance from the outer edge of the core. Image (c) shows that the outer edge of the halo is well defined, and image (1) is taken outside that radius and shows small separated NP smoke clusters. The sample is coated with $6 \mathrm{~nm}$ of carbon to decrease charging.

no apparent attraction to either plate. With the field on, the average lifespan of the LBs was significantly shorter than when the field was off [19].

We conclude that LBs carry a small net charge of either polarity, more often negative than positive. We hypothesize that silicon cores ejected from the side of the lump where electrons enter during the arc carry extra electrons, giving rise to their negative charge (and vice versa for the exit side). The preferential negative charge would then imply that more

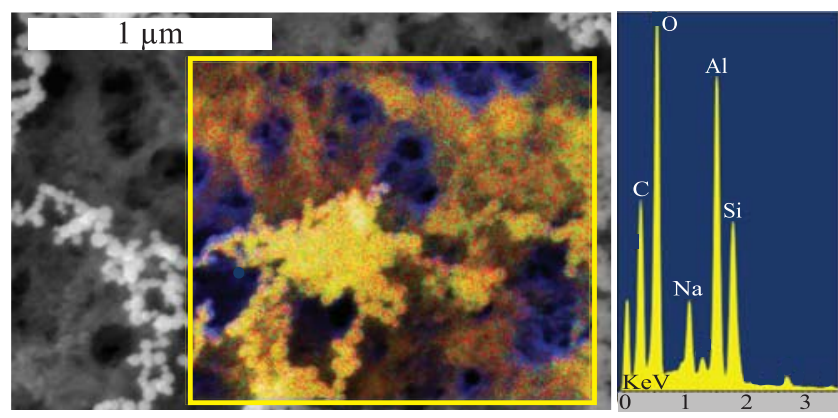

FIG. 5. (Color online) EDS elemental map and spectrum of an LB's NP halo quenched on an Al substrate. Blue regions (dark gray) are Al. Yellow regions are a mixture of $\mathrm{Si}$, green, and $\mathrm{O}$, red. Note that green and red mix to yellow in RGB color space, appearing light gray in print.
LBs are ejected from the side of the lump the arc enters (noting that arc entry side varies since we use an ac welder).

We obtained an estimate of the magnitude of the charge on both positive and negative LBs by tracking 50 LBs' horizontal positions in the field region. Small LBs curve parabolically toward the plate with charge opposite to their own. We fit this curve to the trajectory of a particle undergoing constant horizontal acceleration $\left[x(t)=x_{0}+v_{0 x} t+\frac{1}{2} a_{\mathrm{fit}} t^{2}\right]$ and extract $a_{\text {fit }}$ (see Fig. 6). We assume that LBs have the mass

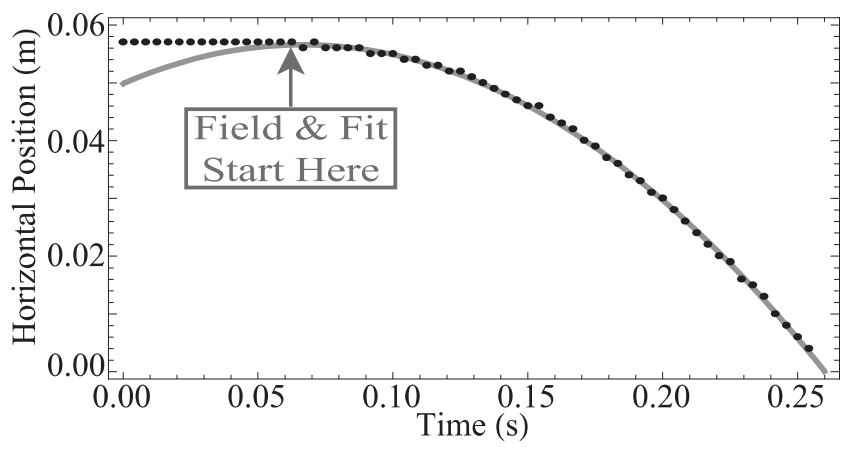

FIG. 6. Horizontal position of a negatively charged LB (black dots) in the capacitor field vs time fit to the trajectory expected (gray line) for a charged particle undergoing constant horizontal acceleration due to the capacitor's electric field. 
TABLE I. Results of tracking $250 \mathrm{LBs}$ traveling in a $45 \pm 5 \mathrm{kV}$ capacitor, for each indicated field status (750 total LBs tracked).

\begin{tabular}{|c|c|c|c|c|c|c|c|}
\hline \multirow[b]{2}{*}{ Field status } & \multirow[b]{2}{*}{ Average LB lifespan (s) } & \multirow[b]{2}{*}{ Average no. of bounces } & \multicolumn{2}{|c|}{$\begin{array}{l}\% \text { quenched } \\
\text { on a plate }\end{array}$} & \multicolumn{2}{|c|}{$\%$ extinguished } & \multirow[b]{2}{*}{$\%$ Not attracted $^{\mathrm{a}}$} \\
\hline & & & Left & Right & Heading left & Heading right & \\
\hline Pointing left & 0.36 & 1.37 & 29.4 & 58.8 & 1.63 & 7.35 & 2.86 \\
\hline Pointing right & 0.39 & 1.64 & 71.6 & 22.0 & 1.60 & 2.00 & 2.80 \\
\hline Off & 0.88 & 5.44 & 20.2 & 23.5 & 3.64 & 3.24 & 49.4 \\
\hline
\end{tabular}

a"\% Not attracted" means the LB quenched while bouncing on the floor between the plates.

$(m)$ of a 1-mm-diameter sphere of liquid silicon $(1.35 \mathrm{mg})$. The magnitude of the charge on the particle is $q_{\mathrm{LB}}=m\left|a_{\mathrm{fit}}\right| d / V$, where $d$ is the plate separation and $V$ is the voltage. Doing so, we find the average charge on positive $\mathrm{LBs}$ to be $10 \pm 7 \mathrm{pC}$ and on negative LBs to be $7 \pm 4 \mathrm{pC}$. There is uncertainty in this value due to the mass estimate, but this mass is consistent with typical quenched LB cores. All 50 particles we measured had to within an order of magnitude the same charge, regardless of polarity (see Fig. 7).

Charge on LB cores may be integral to the binding of the NPs via electrostatic attraction. The NPs should acquire an induced dipole moment in the field of the core and feel a binding force [20]. The radius of the halo would then be proportional to the charge on the core. Net charge on LBs could explain sightings in which ball lighting travels against the direction of wind. In a thunderstorm, the electric field near the earth is smaller than in our capacitor field $(\sim 10 \mathrm{kV} / \mathrm{m}$ compared to our $690 \mathrm{kV} / \mathrm{m})$ [1]. However, we expect the charge imparted to our LBs to be lower than the charge on a natural ball lightning since our current source is smaller by a factor of 100-1000 than lightning currents [2]. Furthermore, we expect the radii of natural ball lightning cores to exceed those of laboratory LBs, giving them larger capacitances. Thus, the cloud-ground potential or other nearby high voltage sources may significantly affect natural ball lightning trajectories via Lorentz forces.

The $100-\mathrm{Hz}$ intensity fluctuations reported in [7] for a natural ball lighting $20 \mathrm{~m}$ from a $50-\mathrm{Hz}, 35-\mathrm{kV}$ power line could be explained by the expansion and contraction of the NP halo with each polarity flip in the field near the line. This is consistent with the hypothesis that the radius of the halo is determined by the field strength.

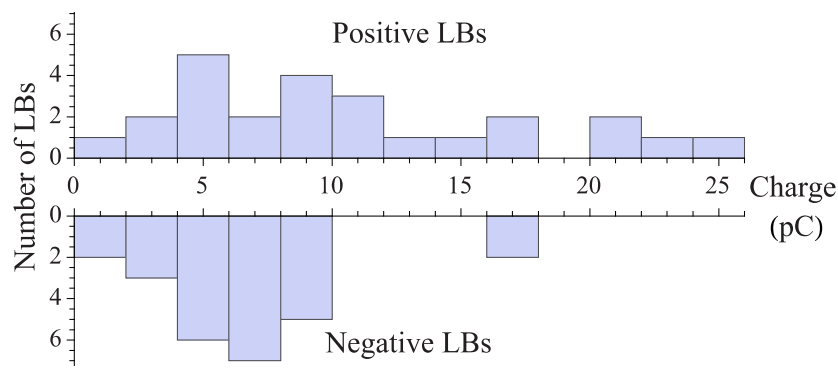

FIG. 7. (Color online) Charge distribution for 50 LBs. Only LBs that touched no objects before striking a plate were measured.
We observed "rocket type bouncing" as described in [10] for LBs bouncing vertically, in which LBs achieve higher jump heights after a succession of lower bounces. We also observed this effect in the horizontal direction, for LBs bouncing between insulating, nonmetal walls several centimeters apart [17]. We do not see this effect on bare metal plates. Interestingly, the effect is enhanced when the horizontal field of the capacitor plates is turned on and an insulator is placed between the aluminum surface and the LBs. We hypothesize that this is because (1) the field increases the likelihood that a charged LB will have a strong impact with a vertical plate and (2) the presence of the insulator inhibits LB quenching. The rocket bouncing seemingly is not due to LB cores charging and flipping polarity with each impact [21].

\section{CONCLUSION}

We produced luminous balls, similar in appearance to natural ball lightning, using lump silicon. We achieved a higher production rate, average diameter, and average lifespan for the balls than previously reported. Our LBs can be convected upward in winds consistent with thunderstorms. We confirmed via microscopy that the structure of our fireballs is in basic agreement with those produced by Ito and Cappelli [14], though we note that there are two distinct rings in the NP halos we produce. The halo's structure is consistent with an electrostatic binding force balancing NP ejection from the core and potentially other fluid mechanical forces, with an inner ring likely defined by the distance at which $\mathrm{SiO}$ fully oxides to $\mathrm{SiO}_{2}$. EDS showed that the LB cores are metallic silicon with a thin oxide layer on the outside, and that the NPs are composed of $\mathrm{SiO}_{2}$.

We measured the net charge of a typical LB to be on the order of picocoulombs, of either positive or negative polarity. We propose that this charge is imparted to the core during its formation, and is integral to the binding of the NPs. If indeed this model describes a class of natural ball lightning, as suggested by recent observation [7], a net charge on the fireballs may explain reported erratic trajectories or intensity fluctuations.

\section{ACKNOWLEDGMENTS}

This research was supported by Reed College as well as the Sanchez Nano-Development Laboratory's Research Sponsored Projects Group at Portland State University. Imaging was performed at the Portland State University Center for Electron Microscopy and Nanofabrication. 
[1] M. Stenhoff, Ball Lightning: An Unsolved Problem in Atmospheric Physics (Plenum, New York, 1999).

[2] S. Singer, The Nature of Ball Lightning (Plenum, New York, 1971).

[3] W. Brand, Der Kugelblitz (Verlag Von Henry Grand, Hamburg, 1923), Vols. 2 and 3.

[4] J. R. McNally, Jr., Report No. ORNL-3938, Oak Ridge National Laboratory, Oak Ridge, 1966.

[5] W. D. Rayle, NASA Technical Note TN D-3188, Washington, DC, 1966.

[6] J. D. Barry, J. Atmos. Terr. Phys. 29, 1095 (1967).

[7] J. Cen, P. Yuan, and S. Xue, Phys. Rev. Lett. 112, 035001 (2014).

[8] J. Abrahamson and J. Dinniss, Nature (London) 403, 519 (2000).

[9] G. S. Paiva, A. C. Pavão, E. Alpes de Vasconcelos, O. Mendes, and E. Felisberto da Silva, Phys. Rev. Lett. 98, 048501 (2007).

[10] K. D. Stephan and N. Massey, J. Atmos. Terr. Phys. 70, 1589 (2008).

[11] T. Ito, T. Tamura, M. A. Cappelli, and S. Hamaguchi, Phys. Rev E 80, 067401 (2009).

[12] G. S. Paiva, J. V. Ferreira, C. C. Bastos, M. V. P. dos Santos, and A. C. Pavao, Sov. Phys. Usp. 53, 209 (2010).

[13] J. Hill, M. Uman, M. Stapleton, D. Jordan, A. Chebaro, and C. Biagi, J. Atmos. Terr. Phys. 72, 913 (2010).

[14] T. Ito and M. A. Cappelli, AIP Advances 2, 012126 (2012).
[15] C. L. Porter, B.A., Reed College, 2013.

[16] J. Abrahamson, Phil. Trans. R. Soc. A 360, 61 (2002).

[17] See Supplemental Material at http://link.aps.org/supplemental/ 10.1103/PhysRevE.90.063102 for video clips of LB experiments.

[18] Our EDS detector was not calibrated with an $\mathrm{SiO}_{2}$ standard for fully quantitative analysis, allowing only rough stoichiometry for $\mathrm{Si}$ and $\mathrm{O}$.

[19] At $45 \mathrm{kV}$, arcing - uncorrelated with LB behavior-occasionally occurred across the edges of the plates. Discharges were infrequent and did not significantly impact our statistical results. No discharges occurred spatially or temporally near LBs used for charge magnitude measurements. To mitigate concerns about possible invisible coronal discharges occurring when no arcing was present, we repeated the experiment at $30 \mathrm{kV}$ with rounded plate edges and saw the same qualitative behavior.

[20] The charge on and electrical conductivity of the NPs is an open question. It is also possible that the LB core could be surrounded by a weakly ionized plasma.

[21] LB cores are capable of charging upon contact with charged capacitor plates after they are extinguished. However, we do not believe that such charging of the live LBs occurs, since we do not observe horizontal rocket bouncing between bare metal plates (only insulating plates). This may indicate that the $\mathrm{SiO}_{2}$ NP halo is insulating, inhibiting charge transfer. 